\title{
Locking-Free Finite Elements for Unilateral Crack Problems in Elasticity
}

\author{
Z. Belhachmi, J.-M. Sac-Epée and S. Tahir ${ }^{1}$ \\ LMAM UMR7122, Université Paul Verlaine de Metz, Ile du Saulcy, 57045 Metz, France
}

\begin{abstract}
We consider mixed and hybrid variational formulations to the linearized elasticity system in domains with cracks. Inequality type conditions are prescribed at the crack faces which results in unilateral contact problems. The variational formulations are extended to the whole domain including the cracks which yields, for each problem, a smooth domain formulation. Mixed finite element methods such as PEERS or BDM methods are designed to avoid locking for nearly incompressible materials in plane elasticity. We study and implement discretizations based on such mixed finite element methods for the smooth domain formulations to the unilateral crack problems. We obtain convergence rates and optimal error estimates and we present some numerical experiments in agreement with the theoretical results.
\end{abstract}

Key words: crack problems, variational inequalities, smooth domain method, mixed finite elements, a priori estimates

AMS subject classification: 65N30,74M15,35J85

\section{Introduction}

We consider the equilibrium problem for a linear elastic body occupying the domain $\Omega_{c}=\Omega \backslash \bar{\Gamma}_{c}$, $\Omega \subset \mathbb{R}^{2}$, with the interior crack $\Gamma_{c}$ and $\partial \Omega=\Gamma_{D} \cup \Gamma_{N}, \Gamma_{D} \neq \emptyset$. Given a volume force $\mathrm{f}: \Omega_{c} \rightarrow \mathbb{R}^{2}$ and a traction $\mathbf{g}: \Gamma_{N} \rightarrow \mathbb{R}^{2}$, find $\mathbf{u}=\left(u_{1}, u_{2}\right)$, and $\sigma=\left(\sigma_{i j}\right), i, j=1,2$, such that

\footnotetext{
${ }^{1}$ Corresponding author. E-mail: belhach@univ-metz.fr
} 


$$
\begin{aligned}
& -\operatorname{div} \sigma=\mathbf{f} \quad \text { in } \Omega_{c}, \\
& C^{-1} \sigma-\varepsilon(\mathbf{u})=0 \quad \text { in } \Omega_{c} \text {, } \\
& \mathbf{u}=0 \quad \text { on } \Gamma_{D}, \\
& \sigma \mathbf{n}=0 \quad \text { on } \Gamma_{N}, \\
& {[\mathbf{u}] \mathbf{n} \geq 0, \quad[\sigma \mathbf{n}]=0, \quad \sigma_{n}[\mathbf{u}]=0 \quad \text { on } \Gamma_{c}} \\
& \sigma_{n} \leq 0, \quad \sigma_{t}=0 \quad \text { on } \Gamma_{c}^{ \pm} .
\end{aligned}
$$

$\mathbf{u}$ is the displacement field and $\sigma$ is the tensor of constraints. $[\mathbf{u}]=\mathbf{u}^{+}-\mathbf{u}^{-}$denotes the jump of the displacement field $\mathbf{u}$ across $\Gamma_{c}$, and the signs \pm indicate the positive and negative directions of the normal $\mathbf{n}$. We have used the following standard notations

$$
\begin{gathered}
\sigma_{n}=\sigma_{i j} \mathbf{n}_{j} \mathbf{n}_{i}, \sigma_{t}=\sigma \mathbf{n}-\sigma_{n} \mathbf{n}=\left\{\sigma_{t}^{i}\right\}_{i=1}^{2}, \sigma \mathbf{n}=\left\{\sigma_{i j} \mathbf{n}_{j}\right\}_{i=1}^{2}, \\
\varepsilon_{i j}(\mathbf{u})=\frac{1}{2}\left(u_{i, j}+u_{j, i}\right), i, j=1,2, \varepsilon(\mathbf{u})=\left(\varepsilon_{i j}\right)_{i, j=1}^{2}, \\
\sigma=\lambda \operatorname{tr}(\varepsilon(\mathbf{u})) \mathbf{I}_{d}+2 \mu \varepsilon(\mathbf{u}) .
\end{gathered}
$$

$\lambda$ and $\mu$ are the Lamé constants. The fourth order tensor $C$ is symmetric and satisfies the ellipticity condition

$$
c_{i j k \ell} \xi_{j i} \xi_{k \ell} \geq c_{0}|\xi|^{2}, \forall \xi_{j i}=\xi_{i j}, c_{0}>0 .
$$

We use the summation convention over repeated indices.

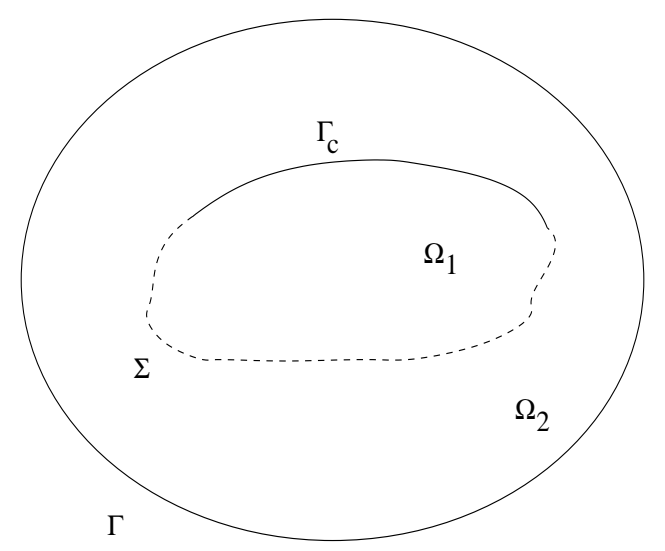

Figure 1: A crack in the reference configuration

The boundary conditions of unilateral type on $\Gamma_{c}^{ \pm}(1.5)-(1.6)$ describe the mutual nonpenetration between the crack faces. The weak formulation of problem (1.1)-(1.6) yields an elliptic variational inequality, we refer the reader for the mathematical analysis of such problems to $[15,25]$ and references therin. Such boundary conditions when prescribed at the external boundary of Lipschitz 
domains are called the frictionless Signorini conditions. They are encountred in many engineering and application domains. Both their mathematical analysis [15, 25] and their numerical analysis $[19,18,17,24,31]$ and more recently $[5,7,3,6,21,14,26,27,22]$ have been widely studied but still remain a source of many challenging problems. The unilateral crack problems are a particular class where the Signorini conditions are prescribed at the crack faces $[23,4,29,30]$.

The mixed variational formulation to problem (1.1)-(1.6) in displacement-stress fields could be extended to the whole domain including the crack $[23,4,30]$. Therefore, with this extended formulation, we work in the Lipschitzian domain and the difficulties due to the presence of the crack as geometric object are reduced. For instance this means more flexibility for the meshes and the use of standard tools of the numerical analysis in Lipschitzian domains.

In another hand, for nearly incompressible materials, i.e. when $\lambda \rightarrow+\infty$, a numerical locking appears empeaching the convergence of the standard finite element approximation of the displacement. It is well known that ressorting to the mixed finite element eliminates the locking phenomenon. Thus, the objective of the finite element discretizations that we propose in this paper is twofold: providing an efficient tool to solve unilateral crack problems in the linear elasticity with the smooth domain formulation and deriving locking-free methods to solve such problems.

The outline of the paper is as follows: In Section 2 we establish the variational formulation and show that it yields a well posed problem. Section 3 is devoted to the description of the discretization methods and the analysis of the discrete problems. The error estimates are established in Section 4. In Section 5 we give some implementation details and we present some numerical experiments to show the efficiency of the approach.

\section{Variational formulation}

Stable mixed finite element for the elasticity problem are usually obtained by relaxing the symmetry of the stress tensor $[28,10]$ which leads to the use of the so-called Hellinger-Reissner modified functional of the elasticity system. This yields to modify the elasticity system (1.1)(1.6) as follows: We seek the displacement $\mathbf{u}: \Omega \rightarrow \mathbb{R}^{2}$, the stress field $\sigma: \Omega \rightarrow \mathbb{R}^{2}$ and $\gamma: \Omega \rightarrow M_{\text {skew }}^{2 \times 2}:=\left\{\eta \in \mathbb{R}^{2 \times 2}: \eta+\eta^{t}=0\right\}$ such that

$$
\begin{gathered}
C \sigma-\nabla \mathbf{u}+\gamma=0 \text { in } \Omega_{c}, \\
\sigma-\sigma^{T}=0 \text { in } \Omega_{c},
\end{gathered}
$$

in place of (1.2) hold. We introduce the functional space

$$
X\left(\Omega_{c}\right)=\left\{\sigma \in L^{2}\left(\Omega_{c} ; \mathbb{R}^{2 \times 2}\right), \operatorname{div} \sigma \in L^{2}\left(\Omega_{c} ; \mathbb{R}^{2}\right), \sigma \nu=0 \text { on } \Gamma_{N}\right\}
$$

equipped with the norm

$$
\|\sigma\|_{X\left(\Omega_{c}\right)}=\left(\|\sigma\|_{L^{2}\left(\Omega_{c} ; \mathbb{R}^{2 \times 2}\right)}^{2}+\|\operatorname{div} \sigma\|_{L^{2}\left(\Omega_{c} ; \mathbb{R}^{2}\right)}^{2}\right)^{\frac{1}{2}},
$$


and the closed convex set

$$
K\left(\Omega_{c}\right)=\left\{\sigma \in X\left(\Omega_{c}\right),[\sigma \nu]=0 \text { on } \Gamma_{c}, \sigma_{t}=0 \text { on } \Gamma_{c}, \sigma_{\nu} \leq 0 \text { on } \Gamma_{c}^{ \pm}\right\} .
$$

Define $V\left(\Omega_{c}\right)=L^{2}\left(\Omega_{c}, \mathbb{R}^{2}\right)$, and introduce $W\left(\Omega_{c}\right)=L^{2}\left(\Omega_{c}, M_{\text {skew }}^{2 \times 2}\right)$.

The mixed variational formulation of the modified system in $\Omega_{c}$ reads:

Find $(\sigma, \mathbf{u}, \gamma) \in K\left(\Omega_{c}\right) \times V\left(\Omega_{c}\right) \times W\left(\Omega_{c}\right)$ such that

$$
\left\{\begin{array}{l}
a(\sigma, \tau-\sigma)+b(\tau-\sigma ; \mathbf{u}, \gamma) \geq 0, \quad \tau \in K\left(\Omega_{c}\right) \\
b(\sigma ; \mathbf{v}, \eta)=-L(\mathbf{v}), \quad(\mathbf{v}, \eta) \in \mathbf{V}\left(\Omega_{c}\right) \times W\left(\Omega_{c}\right)
\end{array}\right.
$$

with

$$
\left\{\begin{array}{l}
a(\sigma, \tau)=\left(C^{-1} \sigma, \tau\right)=\int_{\Omega_{c}} C^{-1} \sigma: \tau d x, \\
b(\sigma ; \mathbf{u}, \gamma)=(\operatorname{div} \sigma, \mathbf{u})+(\sigma, \gamma)=\int_{\Omega_{c}} \operatorname{div} \sigma \cdot \mathbf{u} d x+\int_{\Omega_{c}} \sigma: \gamma d x, \\
L(\mathbf{v})=(\mathbf{f}, \mathbf{v})=\int_{\Omega_{c}} \mathbf{f} \cdot \mathbf{v} d x .
\end{array}\right.
$$

The antisymmetric part of tensor $\tau$ will be denoted by as $(\tau)=\tau_{2,1}-\tau_{1,2}$.

We define the functional space

$$
Z\left(\Omega_{c}\right)=\left\{\sigma \in X\left(\Omega_{c}\right) ; b(\tau ; \mathbf{v}, \eta)=0, \forall(\mathbf{v}, \eta) \in V\left(\Omega_{c}\right) \times W\left(\Omega_{c}\right)\right\},
$$

and we introduce the subset of $K\left(\Omega_{c}\right)$ defined by

$$
\chi=\left\{\tau \in K\left(\Omega_{c}\right) ;-\tau \in K\left(\Omega_{c}\right)\right\} .
$$

The following ellipticity condition holds:

$$
\forall \tau \in Z\left(\Omega_{c}\right), a(\tau, \tau) \geq \alpha\|\tau\|_{X\left(\Omega_{c}\right)}^{2},
$$

as well as the Brezzi-Babuska inf-sup condition [2], there exists a constant $\beta \geq 0$ such that

$$
\forall(\mathbf{v}, \eta) \in \mathbf{V}\left(\Omega_{c}\right) \times W\left(\Omega_{c}\right), \sup _{\tau \in \chi} \frac{b(\tau ; \mathbf{v}, \eta)}{\|\tau\|_{X\left(\Omega_{c}\right)}} \geq \beta\|(\mathbf{v}, \eta)\|_{V\left(\Omega_{c}\right) \times W\left(\Omega_{c}\right)},
$$

with

$$
\|(\mathbf{v}, \eta)\|_{V\left(\Omega_{c}\right) \times W\left(\Omega_{c}\right)}=\|v\|_{L^{2}\left(\Omega_{c} ; \mathbb{R}^{2}\right)}+\|\eta\|_{L^{2}\left(\Omega_{c} ; \mathbb{R}^{2,2}\right)} .
$$

This properties yield the following result (see [29] for details)

Proposition 1. There exists a unique solution $(\sigma, \boldsymbol{u}, \gamma) \in K\left(\Omega_{c}\right) \times V\left(\Omega_{c}\right) \times W\left(\Omega_{c}\right)$ of problem (2.3). Moreover, one has

$$
\boldsymbol{u} \in H_{0, \Gamma_{D}}^{1}\left(\Omega_{c}, \mathbb{R}^{2}\right), \operatorname{as}(\gamma)=\operatorname{curl} \boldsymbol{u},
$$

and

$$
\|\sigma\|_{X\left(\Omega_{c}\right)}+\|\boldsymbol{u}\|_{H^{1}\left(\Omega_{c}, \mathbb{R}^{2}\right)} \leq C\|\boldsymbol{f}\|_{L^{2}\left(\Omega_{c}, \mathbb{R}^{2}\right)}
$$




\subsection{The extended (smooth domain) variational formulation}

The constraint corresponding to the jump condition of the stress tensor's normal component across $\Gamma_{c}$ implies that $\sigma \in X(\Omega)$ (see [16]). Then, following [25, 4, 30]) we can suppress this constraint in the definition of $K\left(\Omega_{c}\right)$ by replacing $X\left(\Omega_{c}\right)$ with $X(\Omega)$, so that we can extend the variational formulation to the whole smooth domain $\Omega$. Set

$$
K=K(\Omega)=\left\{\sigma \in X(\Omega), \sigma_{\nu} \leq 0, \sigma_{t}=0 \text { on } \Gamma_{c}\right\},
$$

and denote by $V$ (resp. $W$ ) the space $V(\Omega)$ (resp. $W(\Omega)$ ). The mixed variational formulation extended to $\Omega$ consists in finding $(\sigma, \mathbf{u}, \gamma) \in K \times V \times W$ such that

$$
\left\{\begin{array}{l}
a(\sigma, \tau-\sigma)+b(\tau-\sigma ; \mathbf{u}, \gamma) \geq 0, \quad \tau \in K \\
b(\sigma ; \mathbf{v}, \eta)=-L(\mathbf{v}), \quad(\mathbf{v}, \eta) \in V \times W
\end{array}\right.
$$

where integrals are extended to $\Omega$.

The ellipticity of the bilinear form $a(.,$.$) and the inf-sup condition verified by b(.,$.$) still hold [29].$ The existence and uniqueness of the solution of Problem (2.4) follows as in proposition 1.

Even if the extended formulation results in a similar problem as in (2.3), we only have

$$
\begin{array}{rlrl}
\text { as }(\gamma) & = & \operatorname{curl} \mathbf{u} \text { in } \mathcal{D}^{\prime}\left(\Omega_{c}\right), \\
C \sigma-\nabla \mathbf{u}+\gamma & =0 \quad \text { in } \mathcal{D}^{\prime}\left(\Omega_{c}\right) .
\end{array}
$$

\subsection{Hybrid formulation}

For practical purposes, either one can solve problem (2.4) which leads to solve high-dimension optimization problems, or the hybrid formulation obtained by expressing the constraints on $\Gamma_{c}$ with Lagrange multipiers that we give now.

We introduce

$$
M=H_{00}^{\frac{1}{2}}\left(\Gamma_{c}, \mathbb{R}^{2}\right),
$$

and

$$
M_{+}=\left\{\mu \in H_{00}^{\frac{1}{2}}\left(\Gamma_{c}\right), \mu \geq 0\right\} .
$$

We also define the bilinear forms $d_{t}(.,$.$) , resp. d_{n}(.,$.$) , on X \times M$, resp. on $X \times M_{+}$,

$$
d_{t}\left(\tau, \lambda_{t}\right)=<<\tau_{t}, \mu_{t}>>_{\frac{1}{2}, 00, \Gamma_{c}}
$$

and

$$
d_{n}\left(\tau, \lambda_{n}\right)=<\tau_{n}, \mu_{n}>_{\frac{1}{2}, 00, \Gamma_{c}}
$$

where $<., .>_{\frac{1}{2}, 00, \Gamma_{c}}$ denotes the duality product between $H_{00}^{\frac{1}{2}}\left(\Gamma_{c}\right)$ and its dual space. 


$$
\begin{aligned}
& <<., .>>_{\frac{1}{2}, 00, \Gamma_{c}} \text { is the duality product defined by } \\
& <<\tau_{t}, \varphi>_{\frac{1}{2}, \Gamma_{c}}=<\tau_{t 1}, \varphi_{1}>_{\frac{1}{2}, 00, \Gamma_{c}}+<\tau_{t 2}, \varphi_{2}>_{\frac{1}{2}, 00, \Gamma_{c}}, \quad \forall \varphi=\left(\varphi_{1}, \varphi_{2}\right) \in M, \varphi_{i} \nu_{i}=0 .
\end{aligned}
$$

The hybrid formulation reads:

Find $\left(\sigma, \mathbf{u}, \gamma, \lambda_{t}, \lambda_{n}\right) \in X \times V \times W \times M \times M_{+}$, with $\lambda_{t_{i}} \nu_{i}=0$, such that

$$
\left\{\begin{array}{l}
a(\sigma, \tau)+b(\tau ; \mathbf{u}, \gamma)+d_{t}\left(\tau, \lambda_{t}\right)+d_{n}\left(\tau, \lambda_{n}\right)=0, \quad \forall \tau \in X \\
(b-\sigma ; \mathbf{v}, \eta)=-L(\mathbf{v}), \quad \forall(\mathbf{v}, \eta) \in V \times W \\
d_{t}\left(\sigma, \mu_{t}\right)=0, \quad \forall \mu_{t}=\left(\mu_{1}, \mu_{2}\right) \in M, \mu_{i} \cdot \nu_{i}=0 \\
d_{n}\left(\sigma, \mu_{n}-\lambda_{n}\right) \leq 0, \quad \forall \mu_{n} \in M_{+}
\end{array}\right.
$$

We get ([29])

Proposition 2. there exists a unique solution $\left(\sigma, \boldsymbol{u}, \gamma, \lambda_{t}, \lambda_{n}\right) \in X \times V \times W \times M \times M_{+}$of problem (2.5). Moreover, one has

$$
\text { as }(\gamma)=\text { curl } \boldsymbol{u}, \lambda_{n}=[\boldsymbol{u}] \nu, \text { and } \lambda_{t}=\boldsymbol{u} \text { on } \Gamma_{c} .
$$

\section{Discrete problems}

Now we deal with the discretization of problem (2.4) and (2.5). The discretization is based on PEERS finite element, introduced by Arnold, Brezzi and Douglas in [2], and the low order BDM finite element, introduced by Brezzi, Douglas and Marini [10], and analysed by Stenberg [28]

We denote by $\mathcal{T}_{h}$ a triangulation of $\Omega$ made of elements which are triangles with a maximum size $h$ satisfying the usual admissibility assumption, i.e. the intersection of two different elements is either empty, a vertex, or a whole edge. In addition, $\mathcal{T}_{h}$ is assumed regular, i.e. the ratio of the diameter of any element $T \in \mathcal{T}_{h}$ to the diameter of its largest inscribed ball is bounded by a constant $\sigma$ independent of $T$ and $h$. We will assume that the endpoints of $\Gamma_{c}$ are vertices of the triangulation. The set of nodes on $\Gamma_{c}$ are denoted by $c_{1}=x_{0}, x_{1}, \ldots, x_{I-1}, x_{I}=c_{2}$ and we set $\left.t_{i}=\right] x_{i-1}, x_{i}[$.

Remark 3. We can assume that on the crack $\Gamma_{c}$ we have a 1-D triangulation completely independent of the triangulation $\mathcal{T}_{h}$ which yields a discretization with high flexibility as regards the triangulation step. However, in this case, we may loose the uniform inf-sup condition on the bilinear form $d(.,$.$) . For the PEERS-based discretization this does not affect the error estimates but for$ the BDM elements the non uniform inf-sup condition reduces the convergence rate. Nevertheless, using the stabilization technique as in [21] allows to recover the full accuracy also in the BDMBased discretization. In this work, we will not follow this direction and we will assume that the triangulation on $\Gamma_{c}$ is the trace of the triangulation $\mathcal{T}_{h}$.

For all $T \in \mathcal{T}_{h}$, we denote by $\mathcal{P}_{k}(T)$ the space of polynomials with total degree less than $k$. 


\subsection{The PEERS's finite element}

Let $R T_{0}$ be the Raviart-Thomas space defined as

$$
R T_{0}=\mathcal{P}_{0}^{2}+(x, y) \mathcal{P}_{0}
$$

Recall that on each $T \in \mathcal{T}_{h}$, the associated non-normalized bubble function is

$$
b_{T}(x)=\prod_{i=1}^{3} \lambda_{i}(x), \quad \forall x \in T,
$$

where $\lambda_{i}(i=1, \cdots, 3)$ are the barycentric coordinates on $T$.

We consider $b_{T}$ extended by zero out of $T$, and define the space generated by rot $b_{T}$ :

$$
A=\left\{\operatorname{rot} b_{T} ; T \in \mathcal{T}_{h}\right\},
$$

and we set

$$
B_{0}(T)=\left\{\tau /\left(\tau_{i 1}, \tau_{i 2}\right) \in A ; i=1,2\right\} .
$$

For the approximation of the stress tensor, we take

$$
X_{h}=\left\{\sigma_{h} \in X ; \sigma_{h \mid T} \in R T_{0}(T)^{2} \oplus B_{0}(T), \forall T \in \mathcal{T}_{h}\right\},
$$

and for the displacement, the approximation space is

$$
V_{h}=\left\{\mathbf{v}_{h} \in L^{2}(\Omega)^{2} ; \mathbf{v}_{h \mid T} \in\left(P_{0}(T)\right)^{2}, \forall T \in \mathcal{T}_{h}\right\} .
$$

The definition of the discrete convex cone $K_{h}$ is identical to that of $K$ with obvious adaptation to the discrete case.

We define

$$
\mathcal{W}_{h}=\left\{\gamma_{h} \in W \cap C^{0}\left(\bar{\Omega}, M_{\text {skew }}^{2 \times 2}\right) ; \gamma_{h \mid T} \in P_{1}\left(T ; M_{\text {skew }}^{2 \times 2}\right), \forall T \in \mathcal{T}_{h}\right\} .
$$

Since the approximation of $\sigma_{h}$ is based on the low level Raviart-Thomas finite element, we choose for the Lagrange multiplier space an approximation with the piecewise-constant functions. We set

$$
W_{h}^{0}\left(\Gamma_{c}\right)=\left\{\mu_{h}, \mu_{h \mid t_{i}} \in \mathbb{P}_{0}\left(t_{i}\right), \quad 0 \leq i \leq I-1\right\},
$$

and we define

$$
M_{h}=W_{h}^{0}\left(\Gamma_{c}, \mathbb{R}^{2}\right)
$$

and

$$
M_{h+}=\left\{\mu_{h} \in W_{h}^{0}\left(\Gamma_{c}\right), \mu_{h} \geq 0\right\} .
$$

We define by $\mathcal{M}_{h}$ the convex cone $M_{h} \times M_{h+}$, and $\lambda_{h}=\left(\lambda_{t h}, \lambda_{n h}\right)$. Note that $M_{h+} \subset M_{+}$. We denote by $\mathcal{E}$ the set of edges $e$ of triangles $T \in \mathcal{T}_{h}$ which are internal to $\Omega_{c}$, and by $J(\mathbf{u})$ the jump $\mathbf{u}^{+}-\mathbf{u}^{-}$across $e$. For the discrete problem analysis, we use the following mesh-dependent norms:

$$
\|\sigma\|_{0, h}^{2}=\frac{1}{E^{2}}\left(\|\sigma\|_{0}^{2}+\sum_{e \in \mathcal{E}_{h}} h_{e}\|\sigma \nu\|_{0, e}^{2}\right), \quad \sigma \in X_{h}
$$




$$
\|\mathbf{u}\|_{1, h}^{2}=\sum_{T \in \mathcal{T}_{h}}\|\mathbf{u}\|_{1, T}^{2}+\sum_{e \in \mathcal{E}_{h}} h_{e}^{-1}\|J(\mathbf{u})\|_{0, e}^{2}, \quad \mathbf{u} \in V_{h}
$$

where for brevity we have noted $\|\sigma\|_{0}=\|\sigma\|_{L^{2}\left(\Omega ; \mathbb{R}^{2 \times 2}\right)},\|\sigma \nu\|_{0, e}=\|\sigma \nu\|_{L^{2}\left(e ; \mathbb{R}^{2}\right)}$ and $\|\mathbf{u}\|_{1, T}=$ $\|\mathbf{u}\|_{H^{1}\left(T ; \mathbb{R}^{2}\right)}$.

Remark 4. 1. Norm $\|\cdot\|_{1, h}$ is used for the BDM finite element's analysis. For PEERS's finite element, one have to modify it according to [[57], section 4]. Since we only recall brad lines of Stenberg's paper, we leave out details.

2. for the BDM finite element, we conform to changing introduced by [18] in the original proof of the inf-sup condition.

In other words, we will write the inf-sup condition with the norm $\|\sigma\|_{0, h}$ and $\|(\mathbf{u}, \gamma)\|_{V_{h} \times \mathcal{W}_{h}}^{2}=$ $\|\mathbf{u}\|_{1, h}^{2}+\|\gamma\|_{0}^{2}$.

\subsubsection{The discrete problem}

For the mixed formulation (2.3), the discrete problem reads:

Find $\left(\sigma_{h}, \mathbf{u}_{h}, \gamma_{h}\right) \in K_{h} \times V_{h} \times \mathcal{W}_{h}$ such that

$$
\left\{\begin{array}{l}
a\left(\sigma_{h}, \tau_{h}-\sigma_{h}\right)+b\left(\tau_{h}-\sigma_{h} ; \mathbf{u}_{h}, \gamma_{h}\right) \geq 0, \tau_{h} \in K_{h} \\
b\left(\sigma_{h} ; \mathbf{v}_{h}, \eta_{h}\right)=-L\left(\mathbf{v}_{h}\right), \quad\left(\mathbf{v}_{h}, \eta_{h}\right) \in V_{h} \times \mathcal{W}_{h} .
\end{array}\right.
$$

Note that bilinear form $a(\cdot, \cdot)$ is coercive only on the subspace $Z$. So, with

$$
Z_{h}=\left\{\tau \in X_{h} ; b\left(\tau_{h} ; \mathbf{v}_{h}, \eta_{h}\right)=0, \forall\left(\mathbf{v}_{h}, \eta_{h}\right) \in V_{h} \times \mathcal{W}_{h}\right\}
$$

one has

$$
a(\sigma, \sigma) \geq C\|\sigma\|_{0, h}^{2}, \forall \sigma \in Z_{h}
$$

Moreover, one has ([57])

$$
\exists \beta>0, \sup _{\tau \in X_{h}} \frac{b(\sigma ; \mathbf{v}, \eta)}{\|\tau\|_{X_{h}}} \geq \beta\|(\mathbf{v}, \eta)\|_{\mathbf{v}_{h} \times \mathcal{W}_{h}}, \forall(\mathbf{v}, \eta) \in V_{h} \times \mathcal{W}_{h} .
$$

These two properties and the standard theory of the mixed variational formulations yield

Proposition 5. Problem (3.1) admits a unique solution $\left(\sigma_{h}, \mathbf{u}_{h}, \gamma_{h}\right) \in K_{h} \times V_{h} \times \mathcal{W}_{h}$.

Again, the hybrid formulation can be written as: Find $\left(\sigma_{h}, \mathbf{u}_{h}, \gamma_{h}, \lambda_{t h}, \lambda_{n h}\right) \in X_{h} \times V_{h} \times \mathcal{W}_{h} \times$ $M_{h} \times M_{h+}$, with $\lambda_{t h, i} \nu_{i}=0$, such that

$$
\left\{\begin{array}{l}
a\left(\sigma_{h}, \tau_{h}\right)+b\left(\tau_{h} ; \mathbf{u}_{h}, \gamma_{h}\right)+d_{t}\left(\tau_{h}, \lambda_{t h}\right)+d_{n}\left(\tau_{h}, \lambda_{h}\right)=0, \quad \forall \tau_{h} \in X_{h} \\
b\left(\sigma_{h} ; \mathbf{v}_{h}, \eta_{h}\right)=-L\left(\mathbf{v}_{h}\right), \forall\left(\mathbf{v}_{h}, \eta_{h}\right) \in V_{h} \times \mathcal{W}_{h} \\
d_{t}\left(\sigma_{h}, \mu_{t h}\right)=0, \forall \mu_{t h}=\left(\mu_{t h, 1}, \mu_{t h, 2}\right) \in M_{h}, \quad \mu_{t h, i} \nu_{i}=0 \\
d_{n}\left(\sigma_{h}, \mu_{n h}-\lambda_{n h}\right) \leq 0, \forall \mu_{n h} \in M_{h+},
\end{array}\right.
$$


or

$$
\left\{\begin{array}{l}
a\left(\sigma_{h}, \tau_{h}\right)+c\left(\tau_{h} ;\left(\mathbf{u}_{h}, \gamma_{h}, \lambda_{t h}, \lambda_{n h}\right)\right)=0, \forall \tau_{h} \in X_{h}, \\
c\left(\tau_{h} ;\left(\mathbf{v}_{h}, \eta_{h}, \mu_{t h}, \mu_{n h}\right)\right)=-L\left(\mathbf{v}_{h}\right), \\
\quad \forall\left(\mathbf{v}_{h}, \eta_{h}, \mu_{t h}, \mu_{n h}\right) \in V_{h} \times \mathcal{W}_{h} \times M_{h} \times M_{h+}, \quad \mu_{t h, i} \nu_{i}=0 .
\end{array}\right.
$$

For $\mu_{h} \in W_{h}^{0}\left(\Gamma_{c}\right)$, we introduce the mesh depending norm

$$
\left\|\mu_{h}\right\|_{L}^{2}=\sum_{i=1}^{I-1} h_{t_{i}}\left\|\mu_{h}\right\|_{L^{2}\left(t_{i}\right)}^{2} .
$$

The following result is proved in the (scalar) case of an elastic membrane in [6]:

$$
\exists \delta>0, \sup _{\tau \in X_{h}} \frac{d_{n}\left(\tau, \mu_{n}\right)}{\|\tau\|_{X_{h}}} \geq C\left\|\mu_{n}\right\|_{L} \geq \delta h^{-\frac{1}{2}}\left\|\mu_{n}\right\|_{L^{2}\left(\Gamma_{c}\right)}, \quad \forall \mu_{n} \in W_{h}^{0}\left(\Gamma_{c}\right) .
$$

No difficulty arises when extending to the elasticity system. We can prove a similar result for the bilinear form $d(.,$.$) defined on \left(X_{h} \times V_{h} \times \mathcal{W}_{h}\right) \times W_{h}^{0}\left(\Gamma_{c}\right)^{3}$ by

$$
d((\tau, \mathbf{v}, \eta), \mu)=d_{t}\left((\tau, \mathbf{v}, \eta), \mu_{t}\right)+d_{n}\left((\tau, \mathbf{v}, \eta), \mu_{n}\right), \quad \mu=\left(\mu_{t}, \mu_{n}\right) .
$$

Existence and unicity of solution $\left(\sigma_{h}, \mathbf{u}_{h}, \gamma_{h}\right)$ of (3.1) derive from Proposition (2.1). So, to prove existence and unicity for the saddle point of formulation (3.2), it suffices to check that

$$
\left\{\mu_{h}=\left(\mu_{t h}, \mu_{n h}\right) \in W_{h}^{0}\left(\Gamma_{c}\right)^{3} ; d\left(\left(\tau_{h}, \mathbf{v}_{h}, \eta_{h}\right), \mu_{h}\right)=0, \quad \forall(\tau, \mathbf{v}, \eta) \in X_{h} \times \mathbf{V}_{h} \times \mathcal{W}_{h}\right\}=\{0\},
$$

which is obvious.

Proposition 6. Problem (3.2) admits a unique solution $\left(\sigma_{h}, \mathbf{u}_{h}, \gamma_{h}, \lambda_{t h}, \lambda_{n h}\right) \in X_{h} \times V_{h} \times \mathcal{W}_{h} \times$ $M_{h} \times M_{h+}$.

\subsection{The zero order BDM finite element}

We define

$$
R_{T}=\{v \in V ; v(x, y)=(a, b)+c(-y, x), a, b, c \in \mathbb{R}\} .
$$

For the space of approximation of the constraints we take

$$
X_{h}=\left\{\sigma_{h} \in X ; \sigma_{h}=\sigma_{h}^{1}+\sigma_{h}^{2}+\sigma_{h}^{3}, \sigma_{h \mid T}^{1} \in \mathcal{P}_{1}^{2 \times 2}, \sigma_{h \mid T}^{2} \in b_{T} \nabla R_{T}, \sigma_{h \mid T}^{3} \in B_{0}(T), \forall T \in \mathcal{T}_{h}\right\},
$$

and for the displacement

$$
V_{h}=\left\{\mathbf{v}_{h} \in(\mathcal{C}(\bar{\Omega}))^{2} ; \mathbf{v}_{h \mid T} \in R_{T}, \forall T \in \mathcal{T}_{h}\right\}
$$

For the antisymmetric tensor, take

$$
\mathcal{W}_{h}=\left\{\gamma_{h} \in W ; \gamma_{h \mid T} \in \mathcal{P}_{1}\left(T ; M_{\text {skew }}^{2 \times 2}\right), \forall T \in \mathcal{T}_{h}\right\} .
$$

For the Lagrange multipliers, it is always possible to take piecewise constant functions like in the PEERS element case, but we know ([6,7]) that piecewise affine functions provide better results when the solutions are more regular. We will choose the space $W_{h}^{1}\left(\Gamma_{c}\right)$ to define $M_{h}$ and $M_{h}^{+}$. We do not write the discrete problems which are the same with (3.1) and (3.2). We have 
Proposition 7. The discrete problem correpsonding to the BDM finite elements admits a unique solution $\left(\sigma_{h}, u_{h}, \gamma_{h}, \lambda_{t h}, \lambda_{n h}\right) \in X_{h} \times V_{h} \times W_{h} \times \mathcal{M}_{h}$, with $\mathcal{M}_{h}=M_{h} \times M_{h}^{+}$.

\subsection{Error estimation}

The error analysis is similar for the PEERS or BDM-based discretizations and is peformed in details in [29]. We summarize in this section the steps of the analysis and the main results. Tedious but standard computations yield the following abstract error estimates: let $\left(\sigma ; \mathbf{u}, \gamma, \lambda_{t}, \lambda_{n}\right)$ denote the solution of problem (2.5), and let $\left(\sigma_{h} ; \mathbf{u}_{h}, \gamma_{h}, \lambda_{t h}, \lambda_{n, h}\right)$ be the solution of the discrete problem (3.2) obtained with the PEERS or BDM elements, there exist constants $C_{1}$ and $C_{2}$ independent of $h$, such that

$$
\begin{aligned}
\| & \sigma-\sigma_{h} \|_{L^{2}\left(\Omega, \mathbb{R}^{2,2}\right)} \leq C_{1}\left\{\inf _{\tau_{h} \in \chi_{h}}\left\|\sigma-\tau_{h}\right\|_{L^{2}\left(\Omega, \mathbb{R}^{2,2}\right)}\right. \\
& +\left[-b\left(\left(\sigma-\tau_{h},\left(\mathbf{u}-\mathbf{u}_{h}, \gamma-\gamma_{h}\right)\right)+L\left(\mathbf{u}-\mathbf{v}_{h}\right)-d_{t}\left(\sigma-\tau_{h}, \lambda_{t}-\lambda_{t h}\right)\right.\right. \\
& +d_{t}\left(\sigma-\sigma_{h}, \lambda_{t}-\mu_{t h}\right)+d_{n}\left(\sigma-\sigma_{h}, \lambda_{n}-\mu_{n h}\right) \\
& \left.\left.-d_{n}\left(\sigma-\tau_{h}, \lambda_{n}-\lambda_{n h}\right)-d_{n}\left(\sigma, \lambda_{n}-\mu_{n h}\right)-d_{n}\left(\sigma, \lambda_{n h}\right)-d_{n}\left(\sigma_{h}, \mu_{n h}\right)\right]^{\frac{1}{2}}\right\} .
\end{aligned}
$$

Moreover, in the case of the PEERS elements, we have

$$
\begin{aligned}
\| & \left(\mathbf{u}-\mathbf{u}_{h}, \gamma-\gamma_{h}\right)\left\|_{\mathbf{v}_{h} \times \mathcal{W}_{h}}+\right\| \lambda_{t}-\lambda_{t h}\left\|_{L^{2}\left(\gamma_{c}, \mathbb{R}^{2}\right)}+\right\| \lambda_{n}-\lambda_{n h} \|_{L^{2}\left(\gamma_{c}\right)} \leq \\
& C_{2}\left(\left\|\sigma-\sigma_{h}\right\|_{L^{2}\left(\Omega, \mathbb{R}^{2,2}\right)}+\inf _{\left(\mathbf{v}_{h}, \eta_{h}, \mu_{h t}, \mu_{h n}\right) \in \mathbf{V}_{h} \times \mathcal{W}_{h} \times \mathcal{M}_{h}}\left(\left\|\left(\mathbf{u}-\mathbf{v}_{h}, \gamma-\eta_{h}\right)\right\|_{\mathbf{v}_{h} \times \mathcal{W}_{h}}\right.\right. \\
& \left.+\left\|\lambda_{t}-\mu_{t h}\right\|_{L^{2}\left(\Gamma_{c}, \mathbb{R}^{2}\right)}+\left\|\lambda_{h}-\mu_{n h}\right\|_{L^{2}\left(\Gamma_{c}\right)}\right),
\end{aligned}
$$

and in the case of the BDM elements

$$
\begin{aligned}
& \left\|\quad\left(\mathbf{u}-\mathbf{u}_{h}, \gamma-\gamma_{h}\right)\right\|_{\mathbf{v}_{h} \times \mathcal{W}_{h}}+\left\|\lambda_{t}-\lambda_{t h}\right\|_{H_{00}^{\frac{1}{2}}\left(\Gamma_{c}, \mathbb{R}^{2}\right)}+\left\|\lambda_{h}-\lambda_{n h}\right\|_{H_{00}^{\frac{1}{2}}\left(\Gamma_{c}, \mathbb{R}^{2}\right)} \leq \\
& \quad \mathcal{C}_{2}\left(\left\|\sigma-\sigma_{h}\right\|_{L^{2}\left(\Omega, \mathbb{R}^{2,2}\right)}+\inf _{\left(\mathbf{v}_{h}, \eta_{h}, \mu_{h t}, \mu_{h n}\right) \in \mathbf{V}_{h} \mathcal{W}_{h} \mathcal{M}_{h}}\left(\left\|\left(\mathbf{u}-\mathbf{v}_{h}, \gamma-\eta_{h}\right)\right\|_{\mathbf{v}_{h} \times \mathcal{W}_{h}}\right.\right. \\
& \left.\left.\quad+\left\|\lambda_{t}-\mu_{t h}\right\|_{H_{00}^{\frac{1}{2}}\left(\Gamma_{c}, \mathbb{R}^{2}\right)}+\left\|\lambda_{h}-\mu_{n h}\right\|_{H_{00}^{\frac{1}{2}}\left(\Gamma_{c}, \mathbb{R}^{2}\right)}\right)\right) .
\end{aligned}
$$

In order to bound the terms appearing in these abstract error estimates, we introduce and recall the main properties for some usefull approximation opertors. We consider the projection operator $\pi_{h}^{0}: L^{2}\left(\Gamma_{c}\right) \rightarrow W_{h}^{0}\left(\Gamma_{c}\right)[59,34]$. This operator satisfies the following estimates: For all function $\varphi \in H^{\nu}\left(\Gamma_{c}\right)$, with $\nu=\frac{1}{2}$ or $\nu=1$, there exists a constant $c>0$, independent of $h$, such that

$$
\left\|\varphi-\pi_{h}^{0} \varphi\right\|_{L^{2}\left(\Gamma_{c}\right)} \leq c h^{\nu}\|\varphi\|_{H^{\nu}\left(\Gamma_{c}\right)} .
$$


Moreover, if $\varphi \in L^{2}\left(\Gamma_{c}\right)$, then

$$
\left\|\varphi-\pi_{h}^{0} \varphi\right\|_{H^{-\frac{1}{2}\left(\Gamma_{c}\right)}} \leq c h^{\frac{1}{2}}\left\|\varphi-\pi_{h}^{0} \varphi\right\|_{L^{2}\left(\Gamma_{c}\right)} .
$$

Note that for $\varphi \geq 0$, one has $\pi_{h}^{0} \varphi \in M_{h}^{0}$. Recall the following results [[57], Lemma 3.1]: With $\|\cdot\|_{k}=\|\cdot\|_{H^{k}\left(\Omega, \mathbb{R}^{2,2}\right)}$ one has

$$
\|\sigma\|_{0} \leq\|\sigma\|_{0, h} \leq C\|\sigma\|_{0}, \forall \sigma \in X_{h},
$$

and

$$
\inf _{\tau \in X_{h}}\|\sigma-\tau\|_{0, h} \leq C h^{k+1}\|\sigma\|_{k+1}, \quad \forall \sigma \in H^{k+1}\left(\Omega, \mathbb{R}^{2,2}\right) \cap X .
$$

We have the following estimates (see [29]):

Proposition 8. Let $\left(\sigma ; \mathbf{u}, \gamma, \lambda, \lambda_{n}\right)$ denote the solution of problem (2.5). Suppose that $\mathbf{u} \in H_{\text {loc }}^{2}\left(\Omega_{c}\right)$, $\sigma \in H_{\text {loc }}^{1}\left(\Omega_{c}\right)$ and that div $\sigma \in H^{1}\left(\Omega_{c}\right)$. Let $\left(\sigma_{h} ; \mathbf{u}_{h}, \gamma_{h}, \lambda_{h}, \lambda_{h n}\right)$ be the solution of the discrete problem (3.2) obtained with the PEERS finite elements. We have the following estimate:

$$
\begin{gathered}
\left\|\sigma-\sigma_{h}\right\|_{0} \leq C(\sigma, \mathbf{u})\left(h\left\|\left(\mathbf{u}-\mathbf{u}_{h}, \gamma-\gamma_{h}\right)\right\|_{\mathbf{v}_{h} \times \mathcal{W}_{h}}+\left\|\lambda_{t}-\lambda_{t h}\right\|_{L^{2}\left(\Gamma_{c}, \mathbb{R}^{2}\right)}\right. \\
\left.+\left\|\lambda_{n}-\lambda_{n h}\right\|_{L^{2}\left(\Gamma_{c}\right)}+h\left\|\lambda_{n h}-\lambda_{n}\right\|_{L^{2}\left(\Gamma_{c}\right)}+h^{\frac{3}{2}}\right) \\
\left\|\left(\mathbf{u}-\mathbf{u}_{h}, \gamma-\gamma_{h}\right)\right\|_{\mathbf{v}_{h} \times \mathcal{W}_{h}}+\left\|\lambda_{t}-\lambda_{t h}\right\|_{L^{2}\left(\Gamma_{c}, \mathbb{R}^{2}\right)}+\left\|\lambda_{n}-\lambda_{n h}\right\|_{L^{2}\left(\Gamma_{c}\right)} \leq \\
C\left(h^{-\frac{1}{2}}\left\|\sigma-\sigma_{h}\right\|_{0}+C(\mathbf{u}) h^{\frac{1}{2}}\right) .
\end{gathered}
$$

$C(\mathbf{u}, \sigma)$ is a constant depending on $\|\mathbf{u}\|_{H_{l o c}^{2}\left(\Omega_{c}\right)}$ and $\|\sigma\|_{H_{l o c}^{1}\left(\Omega_{c}\right)^{4}}$.

Assembling the previous estimates, we have

Theorem 9. Let $\left(\sigma ; \mathbf{u}, \gamma, \lambda, \lambda_{n}\right)$ be the solution of problem (2.5). Suppose that $\mathbf{u} \in H_{\text {loc }}^{2}\left(\Omega_{c}\right)$, $\sigma \in H_{\text {loc }}^{1}\left(\Omega_{c}, \mathbb{R}^{2,2}\right)$ and that div $\sigma \in H^{1}\left(\Omega_{c}\right)$. Let $\left(\sigma_{h} ; \mathbf{u}_{h}, \gamma_{h}, \lambda_{h}, \lambda_{h n}\right)$ be the solution of the discrete problem (3.2), with choices for $\mathbf{M}_{h}$ and $M_{h+}$ given for PEERS's finite elements. We have the following convergence rate:

$$
\begin{aligned}
\left\|\sigma-\sigma_{h}\right\|_{L^{2}\left(\Omega, \mathbb{R}^{2 \times 2}\right)}+\left\|\left(\mathbf{u}-\mathbf{u}_{h}, \gamma-\gamma_{h}\right)\right\|_{\mathbf{v}_{h} \times \mathcal{W}_{h}} & +\left\|\lambda_{t}-\lambda_{h t}\right\|_{L^{2}\left(\Gamma_{c}, \mathbb{R}^{2}\right)} \\
& +\left\|\lambda_{n}-\lambda_{h n}\right\|_{L^{2}\left(\Gamma_{c}\right)} \leq C(\mathbf{u}, \sigma) h^{\frac{1}{2}} .
\end{aligned}
$$

$C(\mathbf{u}, \sigma)$ is a constant depending on $\|\mathbf{u}\|_{H_{l o c}^{2}\left(\Omega_{c}\right)}$, $\|\sigma\|_{H_{l o c}\left(\Omega_{c}\right)^{4}}$ and $\|\operatorname{div} \sigma\|_{H^{1}\left(\Omega_{c}\right)^{2}}$.

Similarly, we obtain for the BDM finite elements:

Proposition 10. Let $\left(\sigma ; \mathbf{u}, \gamma, \lambda, \lambda_{n}\right)$ denote the solution of problem (2.5). Suppose that $\mathbf{u} \in$ $H_{\text {loc }}^{2}\left(\Omega_{c}\right) \sigma \in H_{\text {loc }}^{1}\left(\Omega_{c}, \mathbb{R}^{2,2}\right)$, and that div $\sigma \in H^{1}\left(\Omega_{c}\right)$. Let $\left.\left(\sigma_{h} ; \mathbf{u}_{h}, \gamma_{h}, \lambda_{t h}, \lambda_{n h}\right)\right)$ be the solution of the discrete problem (3.2) obtained with the BDM finite elements. We have the following 
estimate:

$$
\begin{gathered}
\left\|\sigma-\sigma_{h}\right\|_{L^{2}\left(\Omega, \mathbb{R}^{2 \times 2}\right)} \leq C(\sigma, \mathbf{u})\left(h\left\|\left(\mathbf{u}-\mathbf{u}_{h}, \gamma-\gamma_{h}\right)\right\|_{\mathbf{v}_{h} \times \mathcal{W}_{h}}+\left\|\lambda_{t}-\lambda_{t h}\right\|_{H_{00}^{\frac{1}{2}}\left(\Gamma_{c}, \mathbb{R}^{2}\right)}\right. \\
\left.+\left\|\lambda_{n}-\lambda_{n h}\right\|_{H_{00}^{\frac{1}{2}\left(\Gamma_{c}\right)}}+h\left\|\lambda_{n h}-\lambda_{n}\right\|_{H_{00}^{\frac{1}{2}}\left(\Gamma_{c}\right)}+h^{\frac{3}{2}}\right), \\
\left\|\left(\mathbf{u}-\mathbf{u}_{h}, \gamma-\gamma_{h}\right)\right\|_{\mathbf{v}_{h} \times \mathcal{W}_{h}}+\left\|\lambda_{t}-\lambda_{t h}\right\|_{H_{00}^{\frac{1}{2}\left(\Gamma_{c}, \mathbb{R}^{2}\right)}}+\left\|\lambda_{n}-\lambda_{n h}\right\|_{H_{00}^{\frac{1}{2}\left(\Gamma_{c}\right)}} \\
\leq C\left(h^{-\frac{1}{2}}\left\|\sigma-\sigma_{h}\right\|_{L^{2}\left(\Omega, \mathbb{R}^{2 \times 2}\right)}+C(\mathbf{u}) h\right) .
\end{gathered}
$$

$C(\mathbf{u}, \sigma)$ is a constant depending on $\|\mathbf{u}\|_{H_{l o c}^{2}\left(\Omega_{c}\right)},\|\sigma\|_{H_{l o c}^{1}\left(\Omega_{c}\right)^{4}}$ and $\|\operatorname{div} \sigma\|_{H^{1}\left(\Omega_{c}\right)^{2}}$.

Theorem 11. Let $\left(\sigma ; \mathbf{u}, \gamma, \lambda, \lambda_{n}\right)$ be the solution of problem (2.5). Suppose that $\mathbf{u} \in H_{\text {loc }}^{2}\left(\Omega_{c}\right)$, $\sigma \in H_{\text {loc }}^{1}\left(\Omega_{c}, \mathbb{R}^{2,2}\right)$ and that div $\sigma \in H^{1}\left(\Omega_{c}\right)$. Let $\left(\sigma_{h} ; \mathbf{u}_{h}, \gamma_{h}, \lambda_{h}, \lambda_{h n}\right)$ be the solution of the discrete problem (2.5), with choices for $\mathbf{M}_{h}$ and $M_{h+}$ given for PEERS's finite elements. We have the following convergence rate:

$$
\begin{aligned}
\left\|\sigma-\sigma_{h}\right\|_{L^{2}\left(\Omega, \mathbb{R}^{2 \times 2}\right)}+\left\|\left(\mathbf{u}-\mathbf{u}_{h}, \gamma-\gamma_{h}\right)\right\|_{\mathbf{v}_{h} \times \mathcal{W}_{h}} & +\left\|\lambda_{t}-\lambda_{h t}\right\|_{H_{00}^{\frac{1}{2}}\left(\Gamma_{c}, \mathbb{R}^{2}\right)} \\
& +\left\|\lambda_{n}-\lambda_{h n}\right\|_{H_{00}^{\frac{1}{2}}\left(\Gamma_{c}\right)} \leq C(\mathbf{u}, \sigma) h^{\frac{3}{4}} .
\end{aligned}
$$

$C(\mathbf{u}, \sigma)$ is a constant depending on $\|\mathbf{u}\|_{H_{l o c}^{2}\left(\Omega_{c}\right)},\|\sigma\|_{H_{l o c}^{1}\left(\Omega_{c}\right)^{4}}$ and $\|\operatorname{div} \sigma\|_{H^{1}\left(\Omega_{c}\right)^{2}}$.

Note that the difference in the rate of convergence between the PEERS and the BDM-based discretization is due to the choice of the Lagrange multipliers spaces (piecewise-constant functions in the first case, and piecewise affine functions in the second one). This difference only appears when Lagrange multipliers are smoother (when cracks are straight lines, for example).

\section{Numerical implementation}

The details of the implementation are given in [29], we will give in this section a bref review of the method. In order to perform the computations, we will use a commonly used method for problems involving the space $H(\operatorname{div}, \Omega)$ which consists in relaxing the continuity of the normal traces of the constraint tensor $[1,28]$. Such a method leads to a higher dimension but sparse linear system. The continuity of normal traces is considered by means of Lagrange multipliers.

With this implementation, the computations are carried out locally on each element and the Lagrange multipliers (for relaxing the continuity) contain additional informations that can be used to get a better approximation of the displacement with post-processing scheme such as the one introduced in [28] which provides a best approximation of $\mathbf{u}$.

For the method, we replace the approximation space $X_{h}$ by space $X_{h}^{*}$, that we define as follows: Let $\mathcal{R}^{*}$ be the space of vectorial functions whose restrictions to $T$ belong to $R T_{0}(T) \oplus B_{0}(T)$. Set

$$
X_{h}^{*}=\left\{\tau_{h} ;\left(\tau_{i 1}, \tau_{i 2}\right) \in \mathcal{R}^{*}, \forall T \in \mathcal{T}_{h}\right\}
$$


Set

$$
X_{h}^{*}=\left\{\tau_{h} \in X ; \tau_{h \mid T} \in X_{h}^{*}(T), \forall T \in \mathcal{T}_{h}\right\}
$$

Let denote by $\mathcal{E}_{h}$ the set of internal edges of $\mathcal{T}_{h}$, we introduce the discrete spaces of Lagrangian multipliers

$$
\begin{gathered}
Q_{h}=\left\{\lambda: \lambda_{h \mid E} \in \mathcal{P}_{0}(E)^{2}, E \subseteq \mathcal{E}\right\} \\
N_{h}=\left\{l: l_{h \mid E} \in \mathcal{P}_{0}(E)^{2}, E \subseteq \Gamma_{N}, \quad l_{h \mid E}=0 \text { for } E \subseteq \Gamma_{D}\right\} .
\end{gathered}
$$

Introducing Lagrange multipliers ensures the continuity of the normal trace of the stress tensor $\sigma$ on the inner boundaries, so that $\sigma \in H(\operatorname{div}, \Omega)$.

The modified system reads: Find $\left(\sigma_{h}, \mathbf{u}_{h}, \gamma_{h}, \lambda_{t h}, \lambda_{n h}, \alpha_{h}, \beta_{h}\right) \in X_{h}^{*} \times \mathbf{V}_{h} \times \mathcal{W}_{h} \times \mathbf{M}_{h} \times M_{h+} \times$ $Q_{h} \times N_{h}$ such that

$$
\begin{cases}a\left(\sigma_{h}, \tau_{h}\right)+b\left(\tau_{h} ; \mathbf{u}_{h}, \gamma_{h}\right) & -\sum_{E \in \mathcal{E}_{h}} \int_{E}\left[\tau_{h} \cdot \nu\right] \alpha_{h} d s+\sum_{E \subseteq \Gamma_{N}} \int_{E} \tau_{h} \cdot \nu \cdot \beta_{h} d s \\ +d_{t}\left(\tau_{h}, \lambda_{t h}\right)+d_{n}\left(\tau_{h}, \lambda_{n}\right) & =0, \quad \forall \tau \in X_{h}^{*} \\ b\left(\sigma_{h} ; \mathbf{v}_{h}, \eta_{h}\right) & =-\left(f, \mathbf{v}_{h}\right), \quad \forall\left(\mathbf{v}_{h}, \eta_{h}\right) \in \mathbf{V}_{h} \times \mathcal{W}_{h}, \\ \sum_{E \in \mathcal{E}_{h}} \int_{E}\left[\sigma_{h} \cdot \nu\right] m_{h} d s & =0, \quad \forall m_{h} \in Q_{h}, \\ \sum_{E \subseteq \Gamma_{N}} \int_{E} \sigma_{h} \cdot \nu \cdot \ell_{h} d s & =0, \quad \forall \ell_{h} \in N_{h}, \\ d_{t}\left(\sigma_{h}, \mu_{t h}\right) & =0, \quad \forall \mu_{t h}=\left(\mu_{t h 1}, \mu_{t h 2}\right) \in \mathbf{M}_{h}, \quad \mu_{h i} \cdot \nu_{h i}=0 \\ d_{n}\left(\sigma_{h}, \mu_{h}-\lambda_{n h}\right) & \leq 0, \quad \forall \mu \in M_{h+},\end{cases}
$$

To solve this discrete problem we observe that $\left(\sigma_{h}, \mathbf{u}_{h}, \gamma_{h}, \lambda_{t h}, \lambda_{n h}\right)$ is the saddle-point of the associated Lagrangien functional. Thus, we will use an Uzawa type algorithm to determine this saddle-point. Let $\mathbf{V}, \mathbf{U}$ denote the vectors with the entries given by the values of the functions $\left(\tau_{h}, \mathbf{v}_{h}, \eta_{h}, m_{h}, \ell_{h}, \mu_{t h}\right)$ and $\left(\sigma_{h}, \mathbf{u}_{h}, \gamma_{h}, \alpha_{h}, \beta_{h}, \lambda_{t h}\right)$, respectively. Let $M$ and $\Lambda$ be the vectors with the entries given by the values of $\mu_{n h}$ and $\lambda_{n h}$, respectively, for the different choices of the space $\mathcal{M}_{h}$ (according to the choice of the PEERS or BDM element). The saddle-point formulation in the finite dimensional setting reads:

Find $(\mathbf{U}, \Lambda)$ defined by the max-min condition

$$
\max _{S M \geq 0}\left(\min _{\mathbf{V}} \frac{1}{2}{ }^{t} \mathbf{V K V}-{ }^{t} \mathbf{V F}+\left({ }^{t} \mathbf{V} \mathbf{L}\right) S M\right)
$$

where $\mathbf{K}$ denotes the stiffness matrix

$$
\left(\begin{array}{llllll}
B & C & D & E & F & H \\
{ }^{t} C & 0 & 0 & 0 & 0 & 0 \\
{ }^{t} D & 0 & 0 & 0 & 0 & 0 \\
{ }^{t} E & 0 & 0 & 0 & 0 & 0 \\
{ }^{t} F & 0 & 0 & 0 & 0 & 0 \\
{ }^{t} H & 0 & 0 & 0 & 0 & 0
\end{array}\right)
$$

with $B, C, D$, are the matrices associated to the bilinear forms $a(.,),. b(.,), E,$.$F the matrices$ associated to the relaxation of the continuity and the Neumann boundary condition and $H$ is associated to $d_{t}(.,$.$) . The matrix L$ is associated to the bilinear form $d_{n}(.,$.$) . \mathbf{F}$ is the vector corresponding to the external loading and the matrix $S$ expresses the sign conditions for the multipliers. 
Let $m$ denote the number of nodes on $\Gamma_{C}$. We define $\left(\psi_{k}\right)_{k}, 1 \leq k \leq m$ to be the finite element basis associated to $W_{h}^{1}\left(\Gamma_{C}\right)$ and $\left(\varphi_{k}\right)_{k}, 1 \leq k \leq m$, to be the finite element basis associated to $W_{h}^{0}\left(\Gamma_{C}\right)$.

Remark 12. Note that $\psi_{1}$, respectively $\psi_{m-1}$, is constant in $t_{0}$, respectively is constant in $t_{m-1}$, and zero elsewhere.

If $M_{h}$ is $=M_{h}^{0}$ or $=M_{h}^{1}$ then $S$ is given by the identity matrix, else $M_{h}=M_{h}^{*}$, and $S_{i j}=$ $\int_{\Gamma_{C}} \psi_{i} \psi_{j} d \tau, 1 \leq i, j \leq m-1$.

The details for the computations of these matrices are given in [29], we just recall briefly the expression of the coupling matrix $\mathbf{L}$ which is defined in the following way

- If $M_{h}=M_{h}^{1}$ or $M_{h}=M_{h}^{*}$, then

$$
(L)_{i j}=\int_{\Gamma_{C}} \psi_{j}\left(\eta_{i} \cdot \mathbf{n}\right) \cdot \mathbf{n} d \tau, 1 \leq i \leq N, 1 \leq j \leq m-1 .
$$

- If $M_{h}=M_{h}^{0}$, then

$$
(L)_{i j}=\int_{\Gamma_{C}} \varphi_{j}\left(\eta_{i} \cdot \mathbf{n}\right) \cdot \mathbf{n} d \tau, 1 \leq i \leq N, 1 \leq j \leq m-1,
$$

where we denote by $N$ the dimension of the space $X_{h}$ in both cases: PEERS or BDM-based discretization and $\left(\eta_{i}\right)_{i}, 1 \leq i \leq N$ a basis of this space. We emphasis that all matrices evaluations are performed at local level (see [29] for details).

The solution $(\mathbf{U}, \Lambda)$ of (4.2) satisfies the saddle-point conditions and we have

$$
\mathbf{U}=\mathbf{K}^{-1}(\mathbf{F}-L S \Lambda)
$$

Therefore, for $\Phi=S \Lambda$, the saddle-point problem (4.2) can be rewritten as a quadratic programming problem

$$
\min _{\Phi \geq 0}\left(\frac{1}{2}{ }^{t} \Phi^{t} L \mathbf{K}^{-1} L \Phi-{ }^{t} \Phi^{t} L \mathbf{K}^{-1} \mathbf{F}+\frac{1}{2}{ }^{t} \mathbf{F} \mathbf{K}^{-1} \mathbf{F}\right)
$$

If $\bar{\Phi}$ is the solution of (4.4) then $\Lambda=S^{-1} \bar{\Phi}$. The solution $\mathbf{U}$ is obtained by solving (4.3).

\subsubsection{Example 1: Elasticity system with $\nu=0.29$}

Let us consider a rectangle $\Omega=(0,4) \times(0,1)$ with the crack $\Gamma_{c}=(0,4) \times\left\{\frac{1}{2}\right\}$. Young's modulus $E$ (resp. the Poisson's ratio $\nu$ ) equals 206900 (resp. 0.29). The external forces are $\mathbf{f}=1$ and $\mathbf{g}=0$. The notation dof stands for the total number of degrees of freedom, while $N_{L^{2}}$ is the $L^{2}$-norm.

In table 1, we represent the $L^{2}$-norm of the relative error of the displacement's first component $u_{1}$, expressed as a function of the total number of degrees of freedom. 


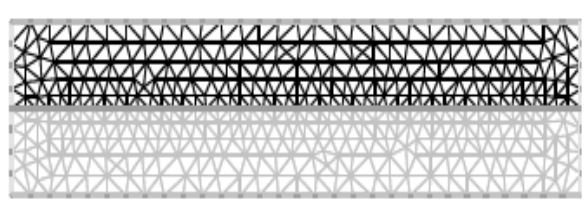

Figure 2: $\Gamma_{c}=(0,4) \times\left\{\frac{1}{2}\right\}$

\begin{tabular}{|l||c|c|c|c|c|}
\hline dof & 83 & 162 & 461 & 819 & 937 \\
\hline$N_{L^{2}}$ & 0.227449873 & 0.0844177121 & 0.0489419458 & 0.0394980417 & 0.0209586395 \\
\hline
\end{tabular}

Table 1: $\left\|u_{1}-u_{1, h}\right\|_{L^{2}(\Omega)}$

\begin{tabular}{|l||c|c|c|c|c|}
\hline dof & 83 & 162 & 461 & 819 & 937 \\
\hline$N_{L^{2}}$ & 0.318122037 & 0.144441467 & 0.0821550301 & 0.0752721434 & 0.0529486423 \\
\hline
\end{tabular}

Table 2: $\left\|u_{2}-u_{2, h}\right\|_{L^{2}(\Omega)}$

\begin{tabular}{|l||c|c|c|c|c|}
\hline$d o f$ & 83 & 162 & 461 & 819 & 937 \\
\hline$N_{L^{2}}$ & 0.425346615 & 0.239523559 & 0.0669139203 & 0.05465566 & 0.0448078919 \\
\hline
\end{tabular}

Table 3: $\left\|\sigma_{t 1}-\sigma_{t 1, h}\right\|_{L^{2}(\Omega)}$

Table 2 represents the $L^{2}$-norm of the relative error of the displacement's second component $u_{2}$, also expressed as a function of the total number of degrees of freedom.

Table 3 represents the $L^{2}$-norm of the relative error of the first constraints tangential component $\sigma_{t 1}$ expressed as a function of the total number of degrees of freedom.

Table 4 represents the $L^{2}$-norm of the relative error of the constraints normal component $\sigma_{\nu}$ expressed as a function of the total number of degrees of freedom.

\begin{tabular}{|l||c|c|c|c|c|}
\hline$d o f$ & 83 & 162 & 461 & 819 & 937 \\
\hline$N_{L^{2}}$ & 0.412398168 & 0.233872393 & 0.0863552882 & 0.0670412243 & 0.0478285353 \\
\hline
\end{tabular}

Table 4: $\left\|\sigma_{\nu}-\sigma_{\nu, h}\right\|_{L^{2}(\Omega)}$ 
Table 1, Table 2, Table 3 and Table 4 summerize results of our computations, where $u_{1}, u_{2}, \sigma_{t 1}$ and $\sigma_{\nu}$ stand for the reference values obtained on the finest mesh. Figure 3, respectively Figure 4 , represents the relative error for the two components of the displacement field, respectively the tangential components of constraints tensor, as a function of the degrees of freedom in log-log scale. The convergence rates for the relative $L^{2}$ error norm of the x-displacement $u_{1}$ is close to 0.81 while those for the $y$-displacement $u_{2}$ is close to 0.65 (Figure 3). On Figure 4, the convergence rate for the first tangential component $\sigma_{t, x}$ is about 0.93 , and for the second one, $\sigma_{t, y}$, is about 0.78 . In both cases, the numerical rates are close to the values predicted for the BDM element. The same holds true with PEERS element.

\subsubsection{Example 2: The nearly incompressible case $\nu=0.49$}

A second test is provided, with an internal crack $\Gamma_{c}=(1,3) \times\left\{\frac{1}{2}\right\}$. The Young's modulus $E$ (resp. the Poisson's ratio $\nu$ ) equals 206900 (resp. 0.49). The external forces are $\mathbf{f}=1$ and $\mathbf{g}=0$.

Figure 5, respectively figure 6 , represents the relative error for the two components of the displacement field, respectively the tangential components of constraints tensor, as a function of the degrees of freedom in log-log scale. The numerical rates of convergence still agree with the theoretical estimates in the nearly incompressible case.

Figure 7 and figure 8, represent the isovalues of $\sigma_{\nu}$ in each case (i.e. with $\nu=0.29$ and 0.49). Both examples considered in this section show the efficiency of the approach particularly for the nearly incompressible case. More realistic examples in the linearized elasticity are under consideration.

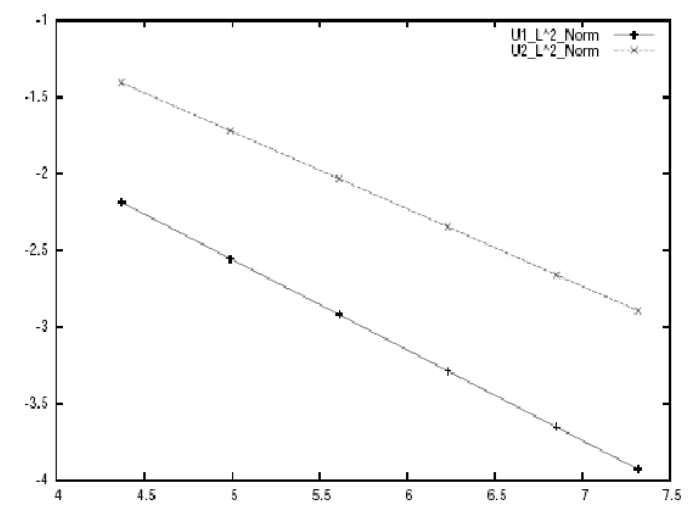

Figure 3: Relative error in the $L^{2}$-norm for displacements as functions of the dof

\section{References}

[1] J. Alberty, C. Carstensen, S. A. Funken, R. Klose. Matlab Implementation of the Finite Element Method in Elasticity. Berichtsreihe des Mathematischen Seminars Kiel, 00-21 (2000). 


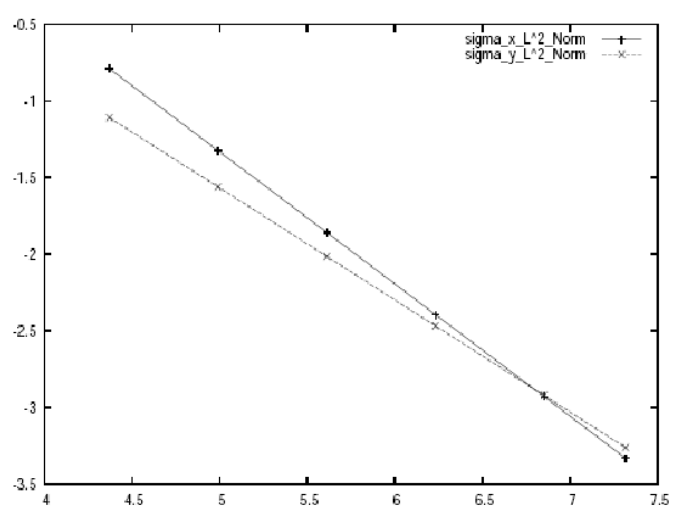

Figure 4: Relative error in the $L^{2}$-norm for constraints as functions of the dof

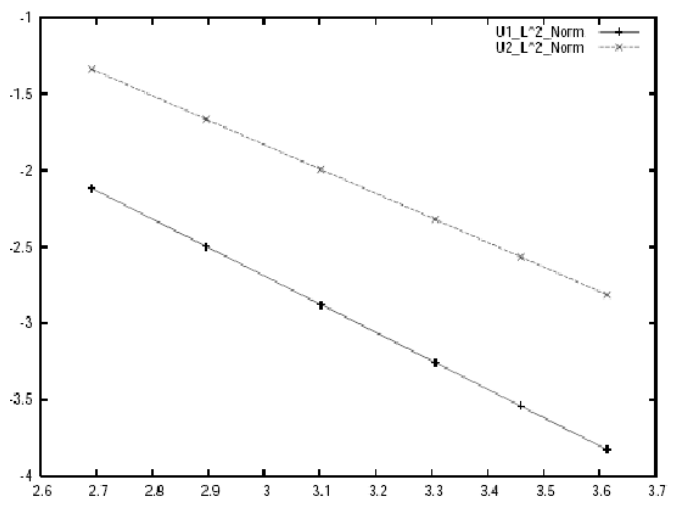

Figure 5: Relative error in the $L^{2}$-norm for displacements as functions of the discretization size

[2] D. N. Arnold, F. Brezzi, J. Douglas. PEERS: A new finite element for plane elasticity Japan J. Appl. Math., No. 1 (1984), 347-367.

[3] Z. Belhachmi, F. Ben Belgacem. Quadratic finite element for Signorini problem. Math. Comp., 72 (2003), No. 241, 83-104.

[4] Z. Belhachmi, J.M. Sac-Epée, J. Sokolowski. Mixed finite element methods for a smooth domain formulation of a crack problem. SIAM J. Numer. Anal., 43 (2005), No. 3, 1295-1320.

[5] F. Ben Belgacem. Numerical simulation of some variational inequalities arisen from unilateral contact problems by finite element method. Siam J. Numer. Anal, 37 (2000),No. 4, 1198-1216.

[6] F. Ben Belgacem, P. Hild, P. Laborde. Extension of the mortar finite element method to a variational inequality modelling unilateral contact. Math. Models Methods Appl. Sci., 9 (1999), No. 2, 287-303.

[7] F. Ben Belgacem, Y. Renard. Hybrid finite element methods for the Signorini problem. Math. Comput., 72 (2003), No. 243, 1117-1145. 


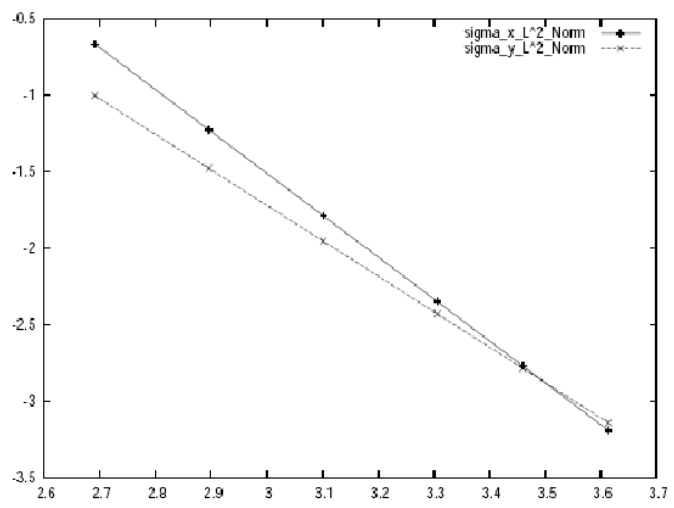

Figure 6: Relative error in the $L^{2}$-norm for constraints as functions of the discretization size

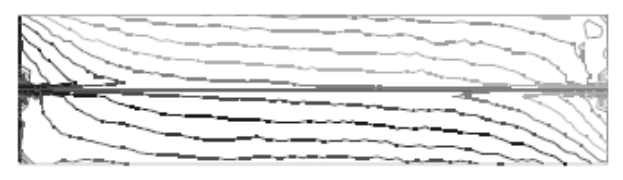

Figure 7: Isovalues for $\sigma_{\nu}$. Example 1

[8] C. Bernardi, V. Girault. A local regularization operator for triangular and quadrilateral finite elements. SIAM. J. Numer. Anal., 35 (1998), No. 5, 1893-1916.

[9] D. Braess, O. Klaas, R. Niekamp, E. Stein, F. Wobschal. Error Indicators For Mixed Finite Elements in 2-dimensional Linear Elasticity. Comput. Methods. Appl. Mech. Engrg., 127 (1995), No. 1-4, 345-356.

[10] F. Brezzi, J. Douglas Jr, L.D. Marini. Two families of mixed finite elements for second order elliptic problems. Numer. Math., 47 (1985), No. 2, 217-235.

[11] F. Brezzi, M. Fortin. Mixed and hybrid finite element methods. Springer Verlag, New York, Springer Series in Computational Mathematics, 15, 1991.

[12] C. Carstensen, G. Dolzmann, S.A. Funken, D.S. Helm. Locking-free adaptive mixed finite element in linear elasticity. Comput. Methods. Appl.Mech. Engrg., 190 (2000), No. 13-14, 1701-1718.

[13] P.G. Ciarlet. Basic Error Estimates for Elliptic Problems. In the Handbook of Numerical Analysis, Vol II, P.G. Ciarlet \& J.-L. Lions eds, North-Holland, (1991), 17-351.

[14] P. Coorevits, P. Hild, K. Lhalouani, T. Sassi. Mixed finite element methods for unilateral problems: convergence analysis and numerical studies. Math. Comp., 71, (2001), No. 237, $1-25$.

[15] G. Duvaut, J.-L. Lions. Les inéquations en mécanique et en physique. Dunod, 1972. 


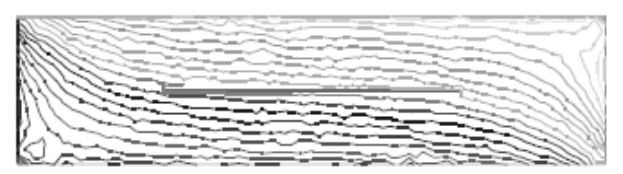

Figure 8: Isovalues for $\sigma_{\nu}$. Example 2

[16] V. Girault, P.-A. Raviart. Finite element methods for the Navier-Stokes equations, Theory and algorithms. Springer-Verlag 1986.

[17] R. Glowinski. Lectures on numerical methods for nonlinear variational problems. Springer, Berlin, 1980.

[18] J. Haslinger, I. Hlaváček. Contact between Elastic Bodies -2.Finite Element Analysis, Aplikace Matematiky, 26 (1981), 263-290.

[19] J. Haslinger, I. Hlaváček, J. Nečas. Numerical Methods for Unilateral Problems in Solid Mechanics, in the Handbook of Numerical Analysis, Vol IV, Part 2, P.G. Ciarlet \& J.-L. Lions eds, North-Holland, 1996.

[20] F. Hecht, O. Pironneau. FreeFem++, www.freefem.org

[21] P. Hild, Y. Renard. An error estimates for the Signorini problem with Coulomb friction approximated by finite elements. Siam J. Numer. Anal., 45 (2007), No. 5, 2012-2031.

[22] S. Hüeber, B.I. Wohlmuth. An optimal a priori error estimates for nonlinear multibody contact problems. SIAM J. Numer. Anal., 43 (2005), No. 1, 156-173

[23] A.M. Khludnev, J. Sokolowski. Smooth domain method for crack problems. Quarterly of Applied Mathematics., 62 (2004), No. 3, 401-422.

[24] N. Kikuchi, J. Oden. Contact problems in elasticity: A study of variational inequalities and finite element methods. SIAM, 1988.

[25] D. Kinderlehrer, G. Stamppachia. An introduction to variational inequalities and their applications, Academic Press, 1980.

[26] K. Lhalouani, T. Sassi. Nonconforming mixed variational formulation and domain decomposition for unilateral problems. East-West J. Numer. Math., 7 (1999), No. 1, 23-30.

[27] L. Slimane, A. Bendali, P. Laborde. Mixed formulations for a class of variational inequalities. M2AN, 38 (2004), 1, 177-201.

[28] R. Stenberg. A family of mixed finite elements for the elasticity problem. Numer. Math., 53 (1988), 5, 513-538. 
[29] S. Tahir. Méthodes d'approximation par éléments finis et analyse a posteriori d'inéquations variationnelles modélisant des problèmes de fissures unilatérales en élasticité linéaire. Ph.D. Thesis, University of Metz, France (2006).

[30] S. Tahir, Z. Belhachmi. Mixed finite elements discretizations of some variational inequalities arising in elasticity problems in domains with cracks. Electron. J. Diff. Eqns., Conference 11 (2004), 33-40.

[31] Z.-H. Zhong. Finite Element Procedures for Contact-Impact Problems. Oxford. University. Press, Oxford 1993. 